\title{
THE UNRELAXED DYNAMICAL STRUCTURE OF THE GALAXY CLUSTER ABELL 85
}

\author{
Heng Yu ${ }^{1,2,3}$, Antonaldo Diaferio ${ }^{2,3}$, Irene Agulli ${ }^{2,3,4,5}$, J. Alfonso L. Aguerri ${ }^{4,5}$, and Paolo Tozzi ${ }^{6,1}$ \\ ${ }^{1}$ Department of Astronomy, Beijing Normal University, Beijing, 100875, Republic of China \\ 2 Dipartimento di Fisica, Università di Torino, Via P. Giuria 1, I-10125 Torino, Italy \\ ${ }^{3}$ Istituto Nazionale di Fisica Nucleare (INFN), Sezione di Torino, Via P. Giuria 1, I-10125 Torino, Italy \\ ${ }_{5}^{4}$ Instituto de Astrofisica de Canarias, C/Via Lactea s/n, E-38200 La Laguna, Tenerife, Spain \\ ${ }^{5}$ Departamento de Astrofisica, Universidad de La Laguna, E-38205 La Laguna, Tenerife, Spain \\ ${ }^{6}$ INAF Osservatorio Astrofisico di Arcetri, Largo E. Fermi, I-50122 Firenze, Italy \\ Received 2016 July 29; accepted 2016 August 26; published 2016 November 4
}

\begin{abstract}
For the first time, we explore the dynamics of the central region of a galaxy cluster within $r_{500} \sim 600 \mathrm{~h}^{-1} \mathrm{kpc}$ from its center by combining optical and X-ray spectroscopy. We use (1) the caustic technique, which identifies the cluster substructures and their galaxy members with optical spectroscopic data, and (2) the X-ray redshift fitting procedure, which estimates the redshift distribution of the intracluster medium (ICM). We use the spatial and redshift distributions of the galaxies and of the X-ray-emitting gas to associate the optical substructures to the X-ray regions. When we apply this approach to Abell 85 (A85), a complex dynamic structure of A85 emerges from our analysis: a galaxy group, with redshift $z=0.0509 \pm 0.0021$ is passing through the cluster center along the line of sight dragging part of the ICM present in the cluster core; two additional groups, at redshift $z=0.0547 \pm 0.0022$ and $z=0.0570 \pm 0.0020$, are going through the cluster in opposite directions, almost perpendicularly to the line of sight, and have substantially perturbed the dynamics of the ICM. An additional group in the outskirts of A85, at redshift $z=0.0561 \pm 0.0023$, is associated with a secondary peak of X-ray emission, at redshift $z=0.0583_{-0.0047}^{+0.0039}$. Although our analysis and results on A85 need to be confirmed by high-resolution spectroscopy, they demonstrate how our new approach can be a powerful tool to constrain the formation history of galaxy clusters by unveiling their central and surrounding structures.
\end{abstract}

Key words: galaxies: clusters: general - galaxies: clusters: individual (Abell 85) - galaxies: clusters: intracluster medium - X-rays: galaxies: clusters

\section{INTRODUCTION}

Optical and X-ray observations of clusters of galaxies and their environs support the scenario where clusters form by accreting matter from their surroundings (Rines et al. 2001; Medezinski et al. 2013; Eckert et al. 2015), as implied by the distribution of galaxies in large and dense galaxy redshift surveys (de Lapparent et al. 1986; Geller \& Huchra 1989; Colless et al. 2001; Abazajian et al. 2003, 2009; Ahn et al. 2014; Hwang et al. 2016) and expected in hierarchical clustering models (Bond et al. 1996; Colberg et al. 2005).

There are various dynamical signatures of this mass accretion process: (i) the presence of substructures in the galaxy density distribution of the cluster on the sky and in redshift space (e.g., Geller \& Beers 1982; Dressler \& Shectman 1988; Ramella et al. 2007; Grillo et al. 2015; Girardi et al. 2015; Balestra et al. 2016, and references therein), (ii) the clumpy distribution either of the hot intracluster medium (ICM) observed in the X-ray band (e.g., Mohr et al. 1993; Kolokotronis et al. 2001; Jeltema et al. 2005; Zhang et al. 2010; Parekh et al. 2015) or (iii) of the dark matter distribution inferred from gravitational lensing effects (e.g., Kneib et al. 1996; Hoekstra et al. 2000; Okabe et al. 2014), and (iv) the presence of diffuse radio emission with elongated or peculiar morphologies (e.g., Girardi et al. 2016, and references therein).

The dynamically unrelaxed state of the cluster might also substantially affect the location of the brightest cluster galaxy (BCG; Lauer et al. 2014), which we expect at the bottom of the gravitational potential well of the cluster (Lin \& Mohr 2004): the BCG can be displaced from the peak of the projected galaxy density distribution (Beers \& Geller 1983), from the peak of the X-ray emission (e.g., Rossetti et al. 2016, and references therein), and from the global redshift of the cluster (Beers et al. 1991; Zitrin et al. 2012).

Identifying cluster substructures and assessing their properties is thus a crucial tool to probe the mass assembly of cosmic structures. Moreover, it can substantially contribute to the investigation of the effect of environment on the evolution of galaxy properties (e.g., Hwang et al. 2012; Pranger et al. 2013, 2014; Hess et al. 2015; Lee et al. 2015; Agulli et al. 2016a; Utsumi et al. 2016).

Substructures are relevant for an additional reason. On the scale of galaxies, significant discrepancies between observations and the cold dark matter model emerge, namely the missing satellite problem, the too-big-to-fail problem, the angular momentum catastrophe, and the cusp-core problem (see, e.g., Del Popolo \& Le Delliou 2016, for a review). A possible solution to these discrepancies is the adoption of additional dark matter components, like warm, self-interacting, or interacting dark matter particles (e.g., Spergel \& Steinhardt 2000; Viel et al. 2013; Bœhm et al. 2014). Although still debated, investigating cluster substructures could in principle distinguish among these dark matter variants. In fact, ordinary matter and different kinds of dark matter behave differently during the collision between cluster components: this different behavior might produce observable differences (e.g., Kahlhoefer et al. 2014; Harvey et al. 2015; Massey et al. 2015; Robertson et al. 2016).

The investigation of the substructure properties requires objective methods to identify cluster substructures. These methods are based on optical or X-ray data, as very briefly reviewed in $\mathrm{Yu}$ et al. (2015). Investigations that combine more 
than one approach are numerous (e.g., Mohr et al. 1996; Bourdin et al. 2011; Guennou et al. 2014; Hwang et al. 2014; Jauzac et al. 2015; Ogrean et al. 2015; Jauzac et al. 2016; Ogrean et al. 2016) and are, in fact, highly desirable as they allow one to infer more robustly the assembly history of the cluster. In addition, and equally important, the combination of different methods can assess the systematic errors of the methods themselves (Geller et al. 2013, 2014).

Here, for the first time, we combine optical and X-ray spectroscopy to associate substructures in the galaxy density distribution with the clumps of the ICM.

With data in the optical band, substructures are difficult to identify even when spectroscopic information is available; in fact, substructures usually contain a limited number of galaxies, and it is extremely difficult to assess the membership of their galaxies, mostly because of the confusion introduced by projection effects. Here we apply the caustic technique, which is based on spectroscopic redshifts (Diaferio \& Geller 1997; Diaferio 1999; Serra et al. 2011), to identify the members of the cluster core and of the cluster substructures and to estimate the redshifts of these structures. Yu et al. (2015) apply this technique to a sample of 150 mock catalogs of clusters extracted from N-body simulations and show that this technique can identify catalogs of substructures that are at least $\sim 60 \%$ complete and contain at most $\sim 50 \%$ spurious substructures. No other available technique appears to perform better on realistic mock cluster catalogs.

When the X-ray emission of clusters is bright enough, the $\mathrm{X}$-ray spectrum may also be used to estimate the redshift of the emitting gas (Yu et al. 2011), although this estimate may suffer from the limited energy resolution of the X-ray detector. Liu et al. $(2015,2016)$ outlined a simple and effective technique to measure the projected X-ray redshifts in different ICM regions and assess the statistical and systematic errors of these redshifts.

By combining the caustic technique and the X-ray redshift fitting, we can infer the motion of the cluster substructures and unveil the complex dynamics of the cluster. We apply this method to a specific cluster: Abell 85 (A85). This cluster is the only system currently available where the sample of galaxy spectra is large enough that we can identify substructures in the same central region covered by the X-ray spectroscopy. We show how, with our approach, we can infer the recent accretion history of A85. It thus appears to be feasible that, when applied to a large sample of clusters with high-quality spectra, our analysis can directly probe the mass assembly of clusters, provide further constraints on hierarchical clustering scenarios on small scales, and eventually probe the properties of the dark matter particles.

In Section 2, we review the estimates of the redshift of A85 with optical and X-ray spectroscopy. Sections 3 and 4 present the optical data we use here and their analysis; the calibration and spectrum fitting of the X-ray data are illustrated in Section 5. The analysis combining optical and X-ray spectroscopy is described in Section 6. Our conclusions and prospects are given in Section 7.

\section{THE GLOBAL REDSHIFT OF A85}

The redshift of a galaxy cluster is usually estimated from the distribution of the redshifts of its member galaxies. The redshift of the brightest galaxy is also adopted when the spectroscopic data are insufficient. However, unavoidably, the estimate of the

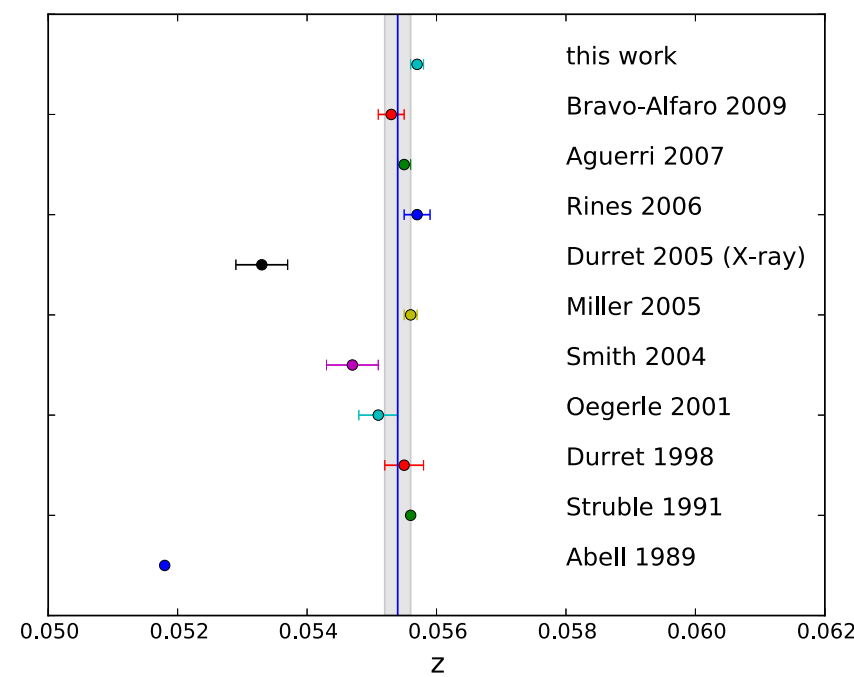

Figure 1. The measured redshift of A85 in the literature. The blue vertical line and the gray shaded area indicate the redshift of the BCG and its error, $z=0.0554 \pm 0.0002$ (Adelman-McCarthy et al. 2008).

cluster redshift is affected by the radial velocity of the substructures of the cluster and therefore by its dynamical state. Therefore, the measurement of the cluster redshift is far from being a trivial issue and is deeply connected with the study of the cluster dynamics and the presence of substructures.

The nearby cluster A85 is a perfect case for a test of our approach combining optical and X-ray spectroscopy. A85 is a rich cluster with a BCG (MCG-02-02-086), X-ray substructures (Tanaka et al. 2010; Schenck et al. 2014; Ichinohe et al. 2015), and filaments (Durret et al. 2003; Boué et al. 2008). The measured redshift $z$ of A85 is slightly different according to different analyses. In early studies, Abell et al. (1989) measure a value of $z=0.0518$ with 59 member galaxies. Struble \& Rood (1991), in their compilation of redshifts for Abell clusters, use 116 galaxies to derive the redshift $z=0.0556$. Durret et al. (1998) perform much deeper observations and measure $z=0.0555 \pm 0.0003$ with 305 optical member galaxies, whose velocities are in the range 13,350-20,000 $\mathrm{km} \mathrm{s}^{-1}$. Oegerle \& Hill (2001) estimate a redshift of $z=0.0551 \pm 0.0003$ with 130 member galaxies. The NOAO Fundamental Plane Survey reports $z=0.0547 \pm 0.0004$ with 58 member galaxies (Smith et al. 2004). Miller et al. (2005) confirm the redshift of Struble $\& \operatorname{Rood}(1991) z=0.0556$ with 82 member galaxies from the SDSS survey Second Data Release (DR2), similar to $z=0.0557$ found with 191 members by Rines \& Diaferio (2006) in the CIRS survey. Aguerri et al. (2007) use 273 redshifts of SDSS-DR4 to derive $z=0.0555 \pm 0.0001$. BravoAlfaro et al. (2009) find 367 member galaxies with a compilation of Abell cluster member galaxies (Andernach et al. 2005), and derive $z=0.0553 \pm 0.0002$.

Figure 1 shows how some of the redshifts listed above significantly differ from the redshift of the brightest galaxy $z=0.0554 \pm 0.0002$ (Adelman-McCarthy et al. 2008); in addition, some differences among the redshifts are larger than their quoted errors: the redshift tends to be underestimated when the sample is not large enough, and, in fact, the disagreement has been recently alleviated by deep redshift surveys like SDSS. Figure 1 shows that the most relevant discrepancy appears between the redshift determined from the X-ray data (Durret et al. 2005) and the optical redshifts, if we 
neglect the early redshift estimate by Abell et al. (1989). In fact, with the XMM-Newton observations, Durret et al. (2005) find that the X-ray redshift of the core of A85, $z=0.0533 \pm$ 0.0004 , is significantly smaller than the average redshift $z=$ 0.0557. Durret et al. (2005) suggest that this discrepancy is originated by the presence of cluster substructures. This suggestion remains unproved.

Here, we use the largest data sample and the newest analysis techniques to unveil the complex structures of A85 that explains the aforementioned discrepancy in the redshift measurements.

\section{OPTICAL SPECTROSCOPIC DATA}

We use the spectroscopic redshift catalog compiled by Agulli et al. (2014, 2016b) based on data from SDSS-DR6 (Adelman-McCarthy et al. 2008), the VIsible Multi-Object Spectrograph at the Very Large Telescope (VI- MOS@VLT, Program 083.A-0962(B), PI R. Sánchez-Janssen, 2009 August), the William Herschel Telescope (WHT), and the NASA/IPAC Extragalactic Database (NED). ${ }^{7}$

Our final sample contains 1603 galaxies: 281 galaxies are brighter than $m_{\mathrm{r}}=17.77$, where $m_{\mathrm{r}}$ is the de-red SDSS-DR6 red-band magnitude, and the sample is $95 \%$ complete to this magnitude; the remaining 1322 galaxies have fainter magnitudes down to $m_{\mathrm{r}}=22$.

We have 241 redshifts from SDSS-DR6 with spectral resolution in the range $R=[1850,2200]$, yielding an uncertainty $\sim 50 \mathrm{~km} \mathrm{~s}^{-1}$ on the Hubble radial velocity derived from the Doppler shift. The 1294 redshifts from VLT/VIMOS and 19 from WHT are derived with low-resolution spectra $(R=180$ and $R=280$ from VLT/VIMOS and WHT, respectively); these resolutions yield an uncertainty $\sim 500 \mathrm{~km} \mathrm{~s}^{-1}$ on the radial velocity, as demonstrated by a number of repeated observations (Agulli et al. 2014). We complete our spectroscopic redshift sample with 49 redshifts from the NED database, for which we do not know the radial velocity uncertainty. To be conservative, we assume $\sigma_{\mathrm{sp}}=500 \mathrm{~km} \mathrm{~s}^{-1}$ as the uncertainty on each individual redshift of our sample.

The large uncertainty on the redshifts and the incompleteness of the galaxy sample at the faint end might affect our substructure identification, as we will discuss in Section 7. However, our aim here is to provide an example of what information we can extract by comparing the redshift distribution of galaxies with the redshift distribution of the $\mathrm{X}$-ray-emitting gas.

Our galaxy sample is the only one currently available that is dense enough in the central region of the cluster that is covered by the X-ray spectroscopy, namely within $r_{500}$, or $\sim 0.6 \mathrm{~h}^{-1} \mathrm{Mpc}$ for A85 (Rines \& Diaferio 2006). The combination of the Chandra fields of A85 probes a box $\sim(0.60 \times 1.2) \mathrm{h}^{-2} \mathrm{Mpc}^{2}$ around the X-ray peak; this area roughly corresponds to the field centered on $[\alpha, \delta]=[10.464623,-9.3699074] \mathrm{deg}$ with 0.1 extension in R.A. $\alpha$ and 0.2 extension in decl. $\delta$ (see Figure 5 below). In this area, we have a unique sample of 243 redshifts, out of which 171 are cluster members, as we will show below.

In addition, the X-ray spectroscopy also yields uncertainties of at least $400 \mathrm{~km} \mathrm{~s}^{-1}$, comparable to the optical uncertainty. Therefore, both optical and X-ray redshifts are insensitive to

\footnotetext{
The redshifts of the cluster members are publicly available in Agulli et al (2016b).
}

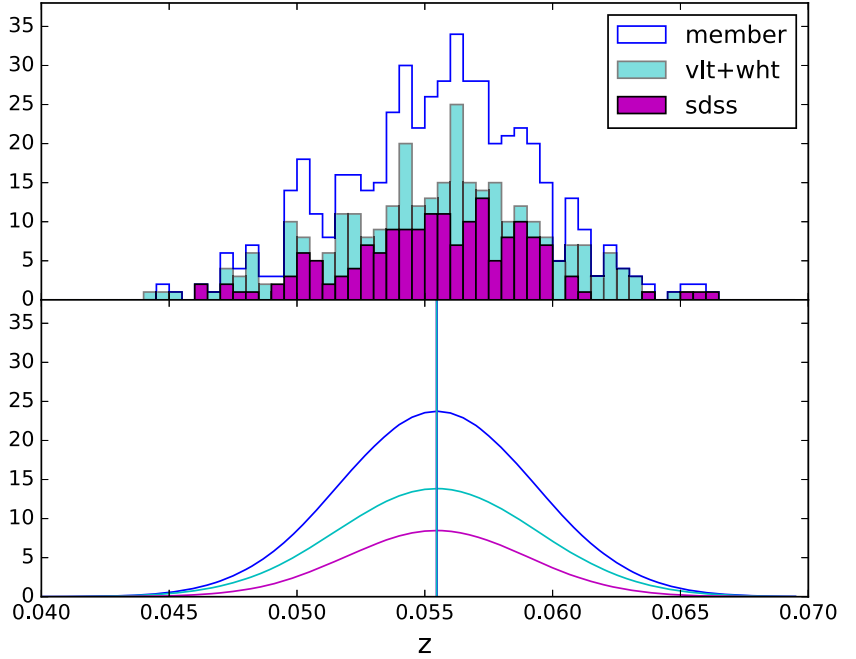

Figure 2. The velocity histogram of the 511 member galaxies in the range 0.04-0.07. The blue open histogram shows the entire distribution. The cyan bars show the 307 galaxies from VLT and WHT. The magenta bars show the 169 galaxies from SDSS. The curves in the lower panel show the Gaussian fit after $3 \sigma$ clipping. The vertical lines indicate the three Gaussian centroids that overlap each other.

substructures with redshift deviations smaller than $\sim 500 \mathrm{~km} \mathrm{~s}^{-1}$.

\section{SUBSTRUCTURES IN THE GALAXY DISTRIBUTION}

There are 515 redshifts in the range $z=[0.04,0.07]$. The distribution of these redshifts $z$ is shown by the open histogram in the upper panel of Figure 2. The application of the traditional $3 \sigma$ clipping procedure only removes 4 galaxies and returns 511 cluster members. The mean redshift and the redshift dispersion of these members are 0.0555 and 0.0038 , respectively. The lower panel of Figure 2 shows that our individual subsamples have comparable average redshifts and velocity dispersions: the VLT + WHT and SDSS galaxy samples have velocity dispersion 1179 and $1060 \mathrm{~km} \mathrm{~s}^{-1}$, respectively.

The histogram is skewed toward the left, suggesting an ongoing merging process that might be one of the reasons for the discrepancy among some of the redshifts reported in the literature.

The caustic method (Diaferio \& Geller 1997; Diaferio 1999; Serra et al. 2011) is based on optical spectroscopic data and has been proved to be a reliable procedure for identifying cluster members (Serra \& Diaferio 2013). It also is a promising tool for identifying substructures ( $Y u$ et al. 2015). It uses the relative binding energy to link galaxies in the field of view and arrange them in a binary tree. By tracing nodes that contain the largest number of galaxies at each bifurcation, we can draw the main branch of the tree. When walking along the main branch from the root to the leaves, the velocity dispersions $\sigma$ of the leaves of each node settle onto a $\sigma$ plateau. The two boundaries of the plateau identify two thresholds that are used to cut the tree at two levels: the first level identifies the cluster members; the second level identifies the cluster substructures (see Serra \& Diaferio 2013; Yu et al. 2015, for further details).

Figure 3 shows the velocity dispersion along the main branch of the binary tree of A85. The 494 galaxies hanging below the first threshold (red solid square in Figure 3 ) are the 


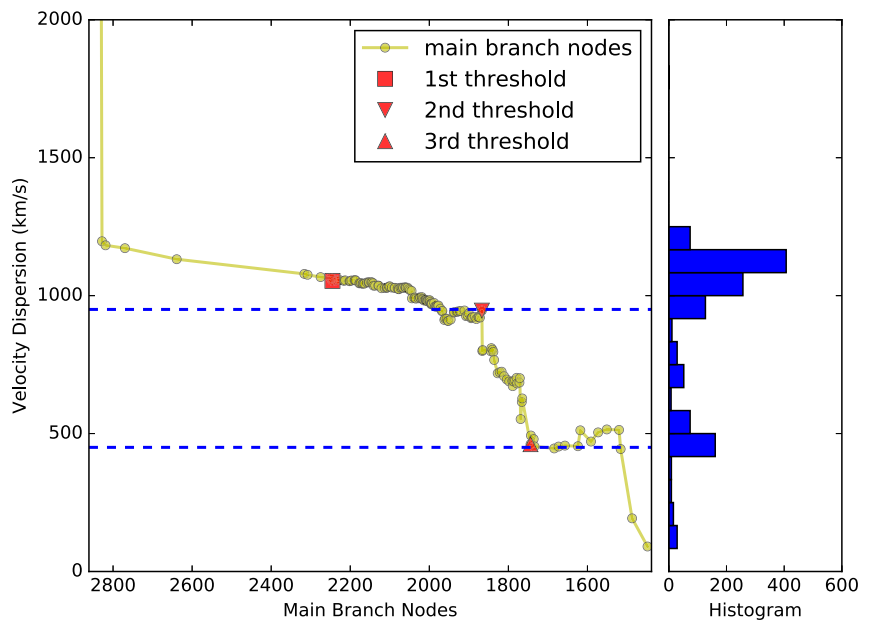

Figure 3. Velocity dispersion of the leaves of each node along the main branch of the binary tree of A85. The histogram in the right panel shows the node numbers in the different velocity dispersion bins. The blue dashed lines indicate the $\sigma$ plateaus. The red symbols are the selected thresholds.

cluster members identified by the $\sigma$ plateau: ${ }^{8}$ their average redshift is $z_{\text {avg }}=0.0554$ and their redshift dispersion $v_{\text {disp }}$ is 0.0035 , as listed in the first row of Table 1 . This velocity dispersion, $1054 \mathrm{~km} \mathrm{~s}^{-1}$, is larger than the velocity dispersion $692_{-45}^{+55} \mathrm{~km} \mathrm{~s}^{-1}$ found by Rines \& Diaferio (2006) based on the 191 galaxy members brighter than $m_{\mathrm{r}}=17.77$ from the SDSSDR4 catalog. These galaxies were identified from the location of the caustics in the redshift diagram extending to $10 \mathrm{~h}^{-1} \mathrm{Mpc}$ from the cluster center. Our sample is slightly different: it covers an area of $3.0 \times 2.6 \mathrm{~h}^{-2} \mathrm{Mpc}^{2}$, and contains all the galaxies on the main branch hanging from the first threshold, thus including those galaxies that are not within the caustics in the redshift diagram and mostly in the tails of the velocity distribution. Our sample limited to the SDSS galaxies within the caustics yields a velocity dispersion consistent with Rines \& Diaferio (2006) result.

Figure 3 shows two plateaus on the main branch: around 950 and $450 \mathrm{~km} \mathrm{~s}^{-1}$. The presence of more than one plateau highlights the complex dynamical state of A85. The first plateau around $950 \mathrm{~km} \mathrm{~s}^{-1}$ is the $\sigma$ plateau automatically identified by the caustic algorithm, whose first boundary identifies the cluster members. The second boundary of the $\sigma$ plateau sets the second threshold which identifies the cluster substructures. $\mathrm{Yu}$ et al. (2015) show that the $\sigma$ plateau automatically located by the algorithm does not always return the most appropriate threshold for the identification of the cluster substructures, especially when the cluster dynamical state is particularly complex. In these cases, as the substructure threshold, we can pick the starting node of a plateau, or alternatively, the first node below the previous plateau. In our case, we choose an additional threshold below $950 \mathrm{~km} \mathrm{~s}^{-1}$, the red triangle around $450 \mathrm{~km} \mathrm{~s}^{-1}$ shown in Figure 3 (the third threshold hereafter). By using these thresholds, we can explore how galaxies populate the cluster at different hierarchical levels. Figure 4 zooms into the central part of the binary tree of our full sample: the upper and lower horizontal lines are the

\footnotetext{
8 The actual list of cluster members provided by the caustic technique is determined by the location of the caustics in the redshift diagram of the cluster (Serra \& Diaferio 2013). For the sake of simplicity, we omit this second step in this analysis: the general conclusions of our analysis remain unaffected.
}

Table 1

Substructures of the Galaxy Distribution

\begin{tabular}{lcccc}
\hline \hline & $N_{\text {gal }}$ & $z_{o}$ & $\sigma_{z}$ & $v_{\text {disp }}\left(\mathrm{km} \mathrm{s}^{-1}\right)$ \\
\hline Cluster & 494 & 0.0554 & 0.0039 & 1054 \\
sub0 & 207 & 0.0546 & 0.0032 & 946 \\
sub1 & 14 & 0.0538 & 0.0020 & 314 \\
sub2 & 12 & 0.0586 & 0.0019 & 255 \\
sub3 & 15 & 0.0550 & 0.0019 & 286 \\
sub4 & 10 & 0.0582 & 0.0022 & 411 \\
\hline sub00 & 38 & 0.0561 & 0.0023 & 461 \\
sub01 & 11 & 0.0570 & 0.0020 & 307 \\
sub02 & 19 & 0.0542 & 0.0022 & 420 \\
sub03 & 18 & 0.0547 & 0.0022 & 423 \\
sub04 & 10 & 0.0509 & 0.0021 & 371 \\
\hline
\end{tabular}

second and third thresholds and individual substructures are identified by different colors.

Table 1 lists the five substructures (from sub0 to sub4) identified by the second threshold located at the end of the plateau at $950 \mathrm{~km} \mathrm{~s}^{-1}$. Table 1 also lists the mean redshift $z_{o}$ of the members of the substructure with its uncertainty $\sigma_{z}=\sqrt{\sigma_{\text {std }}^{2}+\sigma_{\mathrm{sp}}^{2}}$, where $\sigma_{\text {std }}^{2}=\Sigma_{i}\left(z_{i}-z_{o}\right)^{2} /(N-1)$ is the width of the velocity distribution of the structure members, and $\sigma_{\mathrm{sp}}$ is the individual redshift uncertainty. These substructures are shown in the left panel of Figure 5 and are mainly in the cluster outskirts. Figure 5(a) shows that only sub0 is associated to a clump of the X-ray emission. Sub1 to sub4 are surrounding structures. Sub3 and sub4 coincide with the two substructures identified by Aguerri \& Sánchez-Janssen (2010) with the Dressler \& Shectman (1988) method based on SDSS-DR4 data alone. We will not discuss these substructures found with the second threshold, because we do not have X-ray data from their corresponding area on the sky.

The third threshold separates sub0 into individual galaxies and into the five substructures listed in Table 1 from sub00 to sub04 and shown in the right panel of Figure 5. The spatial distribution of these five substructures overlaps with the distribution of the X-ray emission. The radial velocities of sub01 and sub04 show obvious discrepancies with the mean redshift (Figure 6). The substructure sub00, which has the largest number of members (blue circles in the right panel of Figure 5), has redshift $z=0.0561 \pm 0.0023$, slightly larger than the redshift of the system $z_{\mathrm{avg}}=0.0554$, but consistent with the redshift of the X-ray sub-peak ID 12, as we will see below. In fact, sub00 is located south of the X-ray peak, but it is the counterpart of two X-ray sub-peaks. Sub01 (red circles) corresponds to another faint X-ray sub-peak. Sub02 (dark green circles) lies at the location of an X-ray filament (Durret et al. 2003). Sub03 (yellow circles), NE (top left) of the cluster core, includes the BCG. Sub04 (cyan circles) sits on the X-ray peak; however, its redshift $z=0.0509$ is substantially lower than $z_{\text {avg }}=0.0554$ and the BCG redshift, but similar to the redshift of the X-ray peak ID 0 .

Using the photometric data of SDSS-DR6 (AdelmanMcCarthy et al. 2008), we plot the red sequence of our substructures in Figure 7: all the substructures follow the sequence of the main cluster. The fitted red sequence of the cluster is $m_{g}-m_{\mathrm{r}}=-0.0234 m_{\mathrm{r}}+1.2214$. For more details, see Agulli et al. (2016b). The outliers mainly originate from substructure sub02 overlapping the filament in the cluster outskirt. 


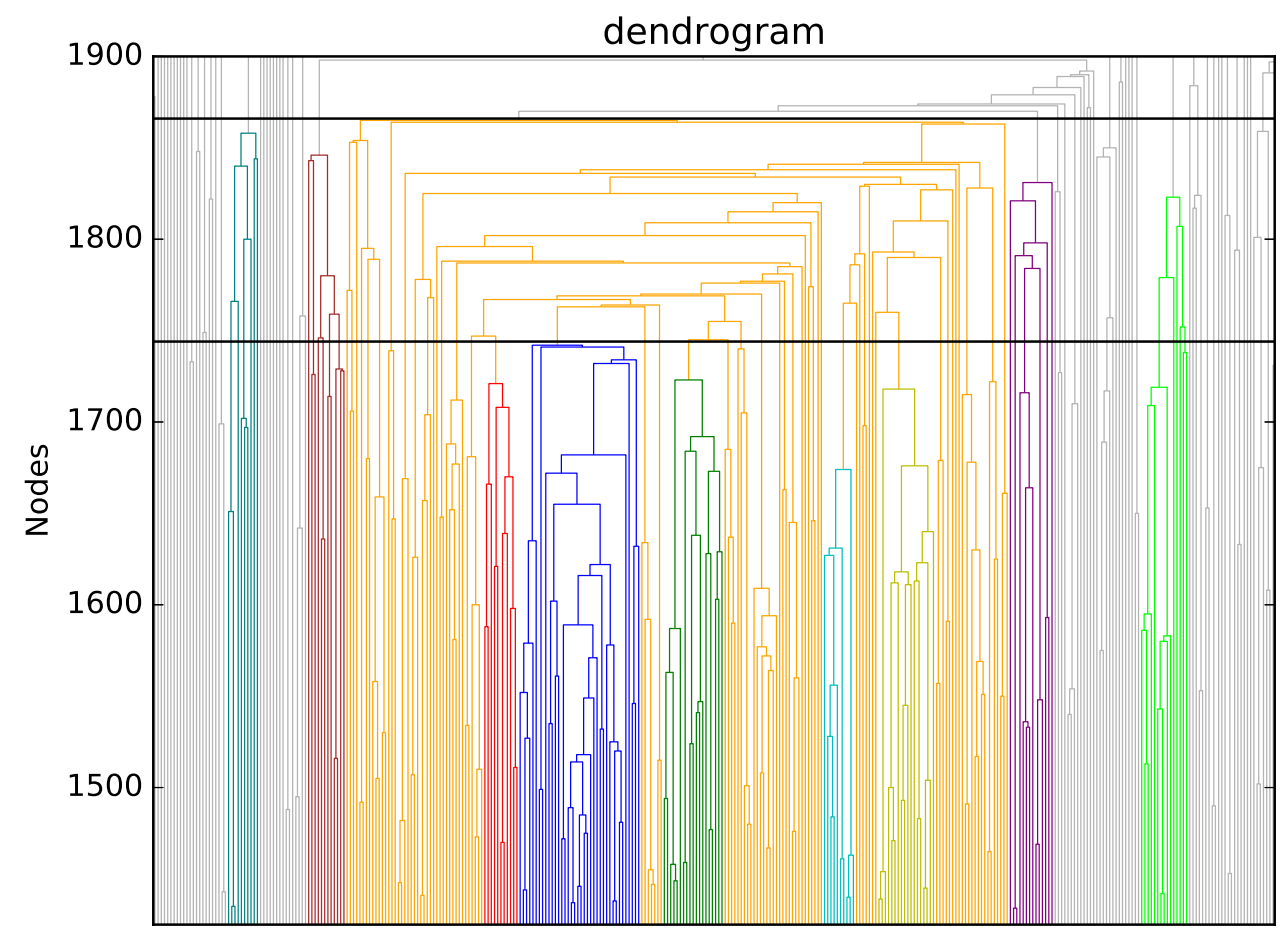

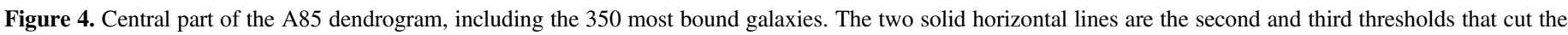

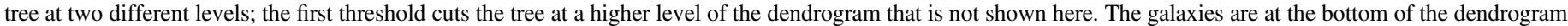

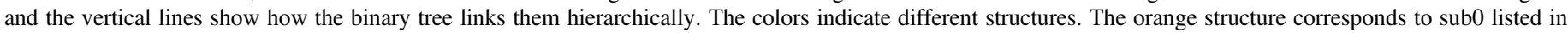

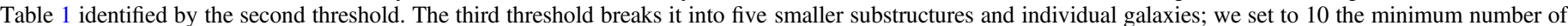
galaxies that defines a substructure.

\section{SUBSTRUCTURES IN THE X-RAY EMISSION}

We use Chandra archived data to estimate the redshift of the cluster and its substructures with the X-ray spectrum fitting procedure. The ObsID 904 is 16 years old and in a different observation mode. The ObsIDs from 4881 to 4888, that contain the A85 field of view, are shallow and offset and do not contain any recognizable structures. To avoid possible calibration errors, we adopt the four most recent ObsIDs, taken in 2013, listed in Table 2.

The selected observations were carried out between 2013 August 9 and 17 in VFAINT mode using the Advanced CCD Imaging Spectrometer (ACIS-I). The data reduction is performed using the latest release of the ciao software (version 4.8) with CALDB 4.7.0. The charge transfer inefficiency (CTI) correction, time-dependent gain adjustment, grade correction, and pixel randomization are applied. We are able to filter efficiently the background events thanks to the VFAINT mode, thus reducing the background by $\sim 25 \%$ in the hard (2.0-10 keV) band. Eventually, we search for high background spikes, and remove them with a $3 \sigma$ clipping. The final exposure times of each ObsID are lower than the nominal exposure time only by a few percent. The level 2 event files obtained in this way are reprojected to match the coordinates of ObsID 15173, and merged into a single event file. The total exposure time of the merged data is $\sim 156.7 \mathrm{ks}$.

Similarly to the analysis performed by Liu et al. (2015) on the Bullet cluster and other clusters (Liu et al. 2016), we apply the contour-binning technique of Sanders (2006) to select regions according to the surface brightness distribution of an extended source. Our goal is to obtain spectra with comparable quality for the measurement of the X-ray redshift. To achieve this goal, we require comparable numbers of net counts in the
$0.5-8 \mathrm{keV}$ band in all the regions. We select a circular region of 530 arcsec including all the visible structures within the chips. This circular region is divided into 24 regions with the condition that the full-band signal-to-noise ratio is larger than 200 in each region. The location and the ID number of these regions are shown in Figure 8. There are two sub-peaks around the cluster: the peak in region 12 (sub-peak A) at the bottom of the image is bright; the peak in region 15 (sub-peak B) at the right of the image is relatively faint.

Because the cluster covers almost the entire X-ray field, we use a nearby region out of our target region to remove the background. All spectra are fitted with Xspec v12.9.0i (Arnaud $1996)$ in the full band $(0.5-8.0 \mathrm{keV})$. To model the X-ray emission, we use double mekal plasma emission models (Mewe et al. 1985, 1986; Kaastra 1992; Liedahl et al. 1995), which include thermal bremsstrahlung and line emission, with abundances measured relative to the solar values in Asplund et al. (2005), where $\mathrm{Fe} / \mathrm{H}=3.6 \times 10^{-5}$. The double-temperature thermal spectrum is helpful to reduce the possible bias in the measurement of the iron line centroid due to the presence of unnoticed thermal structure along the line of sight. Galactic absorption is described by the model tbabs (Wilms et al. 2000). The ICM temperature, the heavy element abundance, the X-ray redshift, and the normalization parameter are all set unconstrained at the same time. The redshifts of the two temperature components are always the same. Considering that there is a large parameter space to explore, we adopt the Markov Chain Monte Carlo method to fit the spectrum. The chain is generated by the Goodman-Weare algorithm (Jonathan Goodman 2010), with 10 walkers, 10,000 burn steps, and a total length of 1,000,000 steps. After the fitting, chains are top-hat filtered according to the following ranges: temperature 


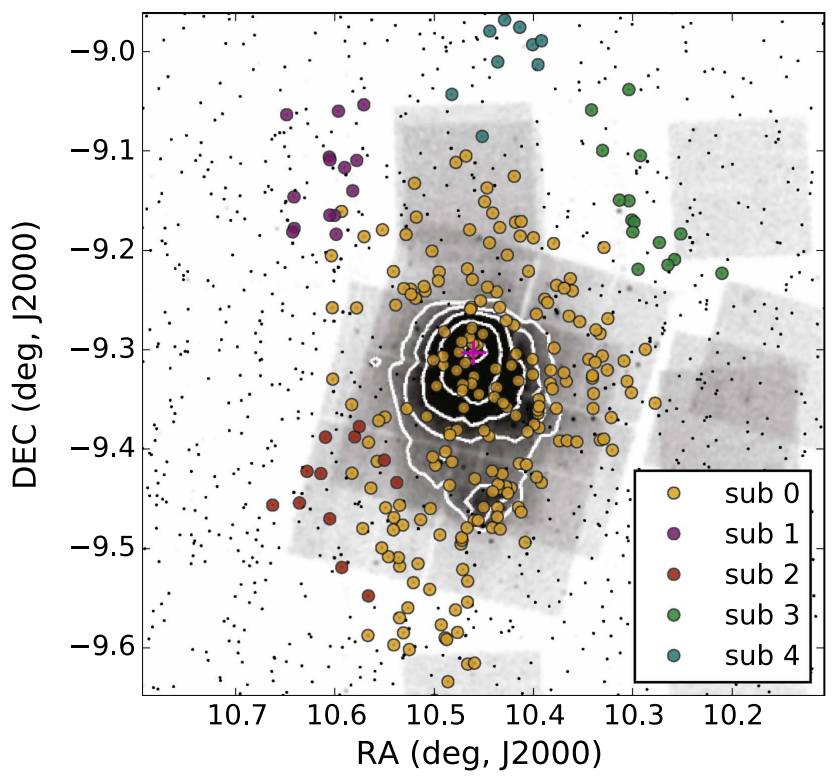

(a) threshold 2

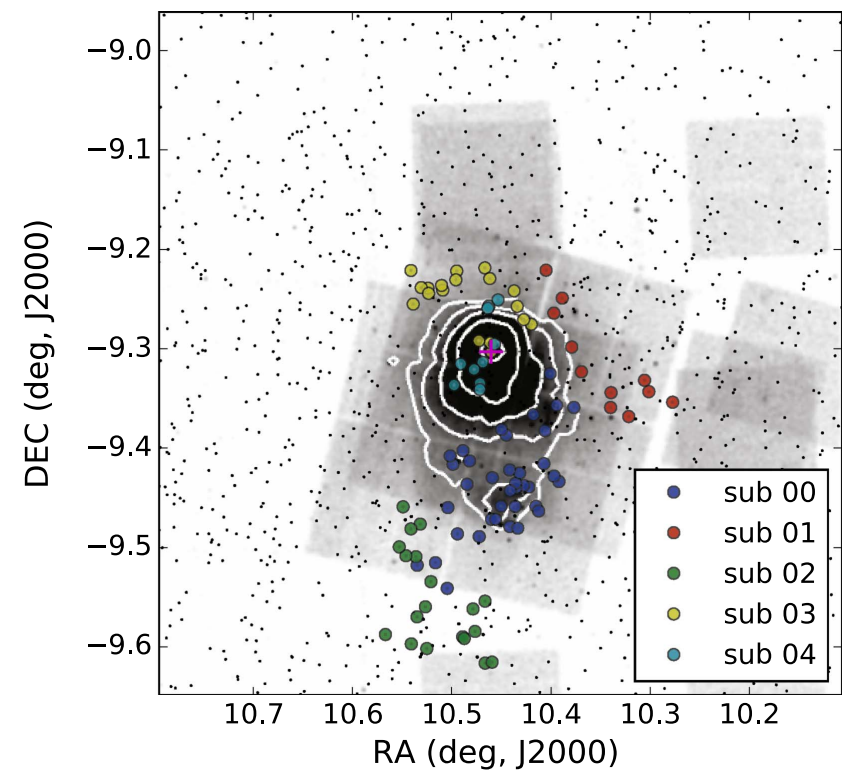

(b) threshold 3

Figure 5. Substructures of the galaxy distributions overlaid on the X-ray image and the X-ray surface brightness contours. Colored solid circles show the galaxies belonging to the individual substructures listed in Table 1 . The position of the BCG is indicated by the purple cross. The color code is the same as in Figure 4 .

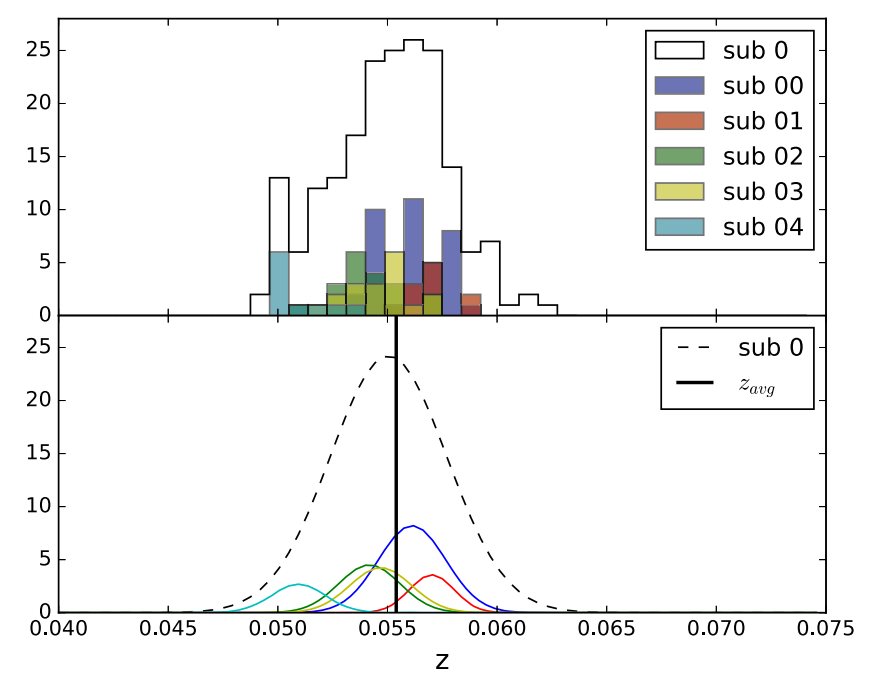

Figure 6. Velocity distributions of the A85 substructures identified with the third threshold. The upper panel shows the velocity histograms. The bottom panel shows the best Gaussian fits. The black vertical line shows the position of the average redshift $z_{\text {avg }}=0.0554$ as a reference.

from 0 to $20 \mathrm{keV}$, metallicity from 0 to 2 , and redshift from 0 to 0.1 . The best fitting parameters and their errors are estimated from these filtered chains.

As we mentioned above, the stacked X-ray image is divided into 24 regions. The fitting results of all the regions are listed in Table 3. Figure 9 shows the redshift deviation $\left(z_{X}-z_{\text {avg }}\right)$ map with respect to the average redshift. Because the X-ray redshift error strongly depends on metallicity, regions ID 3, 4, 5, and 7 in the cluster outskirts of Figure 8 do not have effective redshift values due to their low metallicity $\left(Z / Z_{\odot}<0.15\right)$. Region ID 0 in the cluster center has the most precise redshift because of its high metallicity.

With these measurements, we measure a redshift deviation between the core and the X-ray substructures, which is

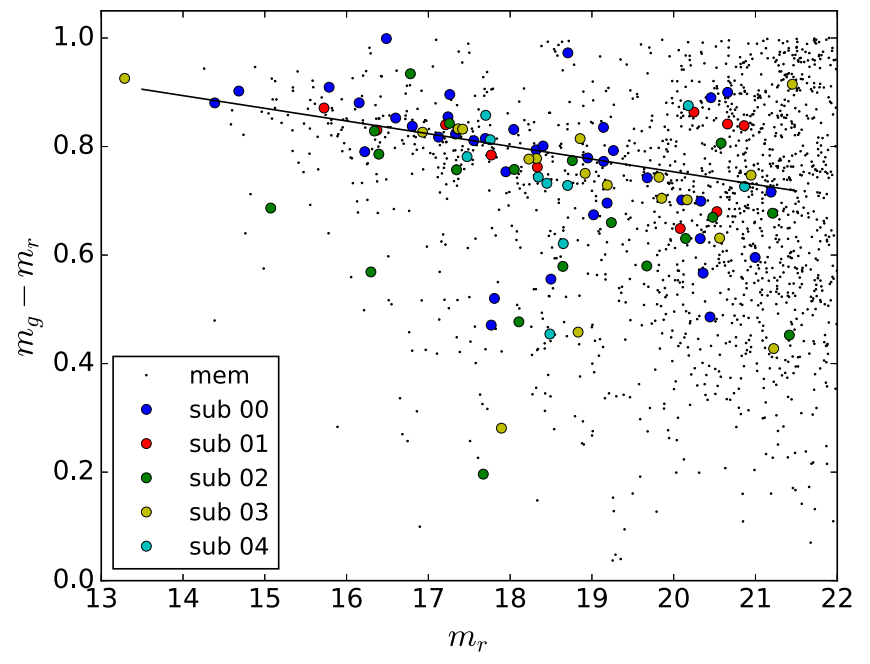

Figure 7. Color-magnitude diagram of the galaxy members of the A85 substructures identified with the third threshold. The color code of the substructure is the same as in Figure 4.

Table 2

List of Chandra Observations

\begin{tabular}{lcccc}
\hline \hline ObsID & Exp $(\mathrm{ks})$ & chips & Mode & Date \\
\hline 15173 & 42.52 & ACIS-I & VFAINT & 2013 Aug 14 \\
15174 & 39.55 & ACIS-I & VFAINT & 2013 Aug 09 \\
16263 & 38.15 & ACIS-I & VFAINT & 2013 Aug 10 \\
16264 & 36.6 & ACIS-I & VFAINT & 2013 Aug 17 \\
\hline
\end{tabular}

consistent with the results of Durret et al. (2005) obtained with $X M M$ data. In addition, for the first time, we can now compare the redshifts of these regions with those of the optical substructures, to investigate the redshift structure of the cluster. 


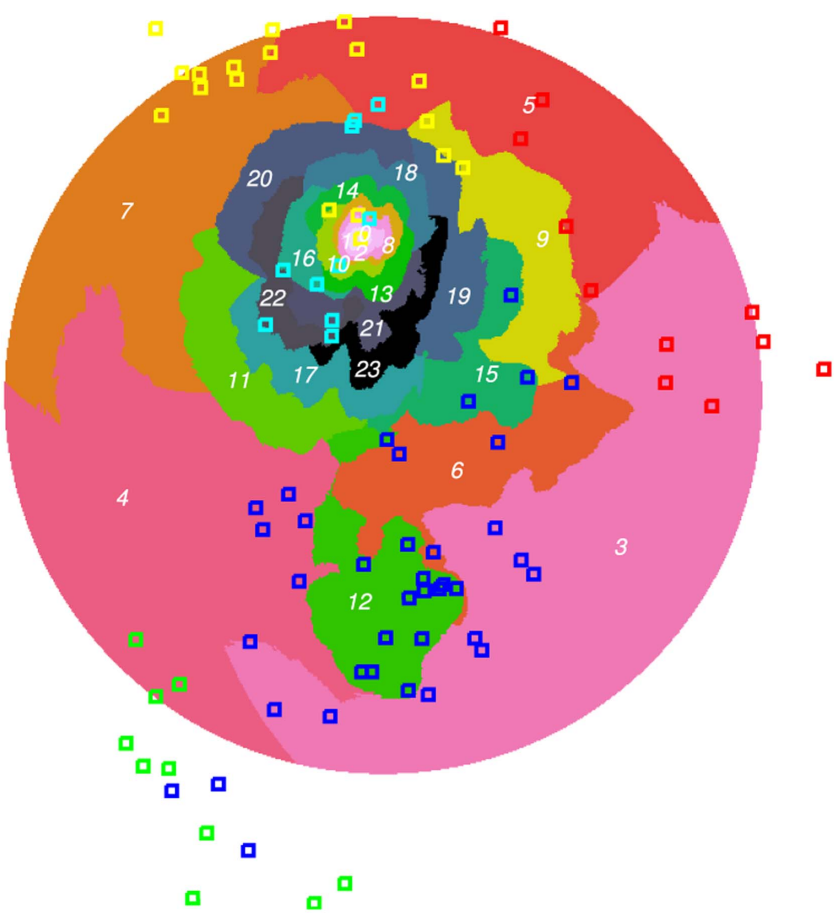

Figure 8. Region division of the $\mathrm{X}$-ray surface brightness with the substructures of the galaxy distribution overlaid. The colors of the galaxies (squares) are the same as in the right panel of Figure 5.

Table 3

X-ray Region Fitting Results

\begin{tabular}{|c|c|c|c|c|c|}
\hline ID & $T_{1}$ & $Z_{1}$ & $T_{2}$ & $Z_{2}$ & $\mathrm{z}$ \\
\hline 0 & $3.47_{-0.18}^{+5.80}$ & $1.16_{-1.06}^{+0.12}$ & $3.47_{-0.48}^{+0.12}$ & $1.10_{-0.08}^{+0.47}$ & $0.0501_{-0.0013}^{+0.0014}$ \\
\hline 1 & $5.01_{-0.74}^{+0.59}$ & $0.46_{-0.21}^{+0.34}$ & $4.29_{-0.37}^{+0.26}$ & $1.16_{-0.34}^{+0.53}$ & $0.0548_{-0.0017}^{+0.0014}$ \\
\hline 2 & $4.98_{-0.42}^{+0.96}$ & $0.61_{-0.42}^{+0.62}$ & $5.14_{-0.51}^{+1.02}$ & $0.57_{-0.41}^{+0.59}$ & $0.0533_{-0.0015}^{+0.0016}$ \\
\hline 3 & $4.48_{-0.31}^{+0.70}$ & $0.04_{-0.03}^{+0.05}$ & $5.52_{-0.93}^{+0.41}$ & $0.12_{-0.05}^{+0.05}$ & $\cdots$ \\
\hline 4 & $6.55_{-0.53}^{+0.74}$ & $0.00_{-0.0}^{+0.0}$ & $6.26_{-0.74}^{+0.97}$ & $0.13_{-0.09}^{+0.095}$ & $\cdots$ \\
\hline 5 & $3.76_{-0.21}^{+0.78}$ & $0.03_{-0.03}^{+0.17}$ & $6.70_{-2.01}^{+0.23}$ & $0.02_{-0.01}^{+0.02}$ & $\ldots$ \\
\hline 6 & $5.85_{-0.58}^{+0.50}$ & $0.19_{-0.06}^{+0.02}$ & $6.05_{-0.58}^{+2.80}$ & $0.17_{-0.06}^{+0.40}$ & $0.0556_{-0.0039}^{+0.005}$ \\
\hline 7 & $6.89_{-0.47}^{+0.73}$ & $0.02_{-0.01}^{+0.02}$ & $6.75_{-0.89}^{+2.58}$ & $0.01_{-0.01}^{+0.01}$ & $\ldots$ \\
\hline 8 & $5.24_{-0.54}^{+0.83}$ & $0.64_{-0.34}^{+0.72}$ & $5.42_{-0.63}^{+0.83}$ & $0.54_{-0.32}^{+0.51}$ & $0.0546_{-0.0014}^{+0.0011}$ \\
\hline 9 & $7.08_{-1.15}^{+1.61}$ & $0.16_{-0.09}^{+0.58}$ & $7.68_{-0.89}^{+0.32}$ & $0.19_{-0.12}^{+0.37}$ & $0.0527_{-0.0043}^{+0.0057}$ \\
\hline 10 & $6.50_{-1.25}^{+3.12}$ & $0.39_{-0.28}^{+0.44}$ & $5.52_{-0.61}^{+0.88}$ & $0.51_{-0.17}^{+0.32}$ & $0.0542_{-0.0018}^{+0.0023}$ \\
\hline 11 & $7.12_{-1.42}^{+2.19}$ & $0.24_{-0.18}^{+0.04}$ & $6.08_{-1.21}^{+3.13}$ & $0.28_{-0.13}^{+0.23}$ & $0.0611_{-0.0037}^{+0.003}$ \\
\hline 12 & $5.64_{-1.87}^{+2.02}$ & $0.27_{-0.21}^{+1.26}$ & $5.28_{-1.45}^{+2.01}$ & $0.35_{-0.27}^{+0.46}$ & $0.0583_{-0.0047}^{+0.0039}$ \\
\hline 13 & $6.15_{-0.69}^{+2.07}$ & $0.59_{-0.16}^{+0.24}$ & $6.51_{-0.49}^{+2.20}$ & $0.37_{-0.30}^{+0.08}$ & $0.0532_{-0.0014}^{+0.0028}$ \\
\hline 14 & $5.95_{-0.72}^{+0.89}$ & $0.41_{-0.29}^{+0.48}$ & $5.43_{-0.73}^{+1.08}$ & $0.73_{-0.62}^{+0.38}$ & $0.0538_{-0.0016}^{+0.0018}$ \\
\hline 15 & $7.16_{-2.89}^{+2.81}$ & $0.22_{-0.12}^{+0.27}$ & $6.68_{-1.30}^{+2.38}$ & $0.40_{-0.13}^{+0.13}$ & $0.0493_{-0.0058}^{+0.0036}$ \\
\hline 16 & $6.63_{-2.03}^{+3.19}$ & $0.88_{-0.33}^{+0.41}$ & $5.62_{-1.06}^{+0.42}$ & $0.41_{-0.10}^{+0.07}$ & $0.0499_{-0.0015}^{+0.0022}$ \\
\hline 17 & $5.78_{-1.86}^{+0.76}$ & $0.40_{-0.21}^{+0.58}$ & $6.86_{-0.69}^{+1.60}$ & $0.35_{-0.10}^{+0.39}$ & $0.0519_{-0.0012}^{+0.0013}$ \\
\hline 18 & $5.93_{-0.55}^{+1.05}$ & $0.32_{-0.25}^{+0.20}$ & $5.66_{-0.80}^{+4.12}$ & $0.91_{-0.53}^{+0.18}$ & $0.0500_{-0.0016}^{+0.0019}$ \\
\hline 19 & $7.39_{-1.23}^{+2.24}$ & $0.20_{-0.15}^{+0.16}$ & $7.08_{-1.34}^{+2.24}$ & $0.36_{-0.16}^{+0.39}$ & $0.0549_{-0.0034}^{+0.0035}$ \\
\hline 20 & $7.21_{-1.80}^{+1.68}$ & $0.25_{-0.17}^{+0.72}$ & $6.75_{-1.42}^{+0.89}$ & $0.22_{-0.16}^{+0.48}$ & $0.0571_{-0.0042}^{+0.0044}$ \\
\hline 21 & $6.75_{-0.73}^{+1.00}$ & $0.49_{-0.12}^{+0.13}$ & $6.07_{-1.25}^{+3.18}$ & $0.37_{-0.17}^{+0.33}$ & $0.0496_{-0.0023}^{+0.0023}$ \\
\hline 22 & $8.71_{-2.40}^{+3.55}$ & $1.93_{-0.13}^{+0.28}$ & $5.92_{-0.39}^{+0.21}$ & $0.31_{-0.08}^{+0.08}$ & $0.0532_{-0.0019}^{+0.0023}$ \\
\hline 23 & $6.97_{-1.27}^{+2.19}$ & $0.13_{-0.10}^{+1.47}$ & $6.98_{-0.85}^{+0.81}$ & $0.41_{-0.37}^{+0.35}$ & $0.0485_{-0.0028}^{+0.0038}$ \\
\hline
\end{tabular}

\section{COMBINING THE OPTICAL AND X-RAY SPECTROSCOPY}

In this section, we combine the $\mathrm{X}$-ray results with the properties of the optical substructures sub00 to sub04, located

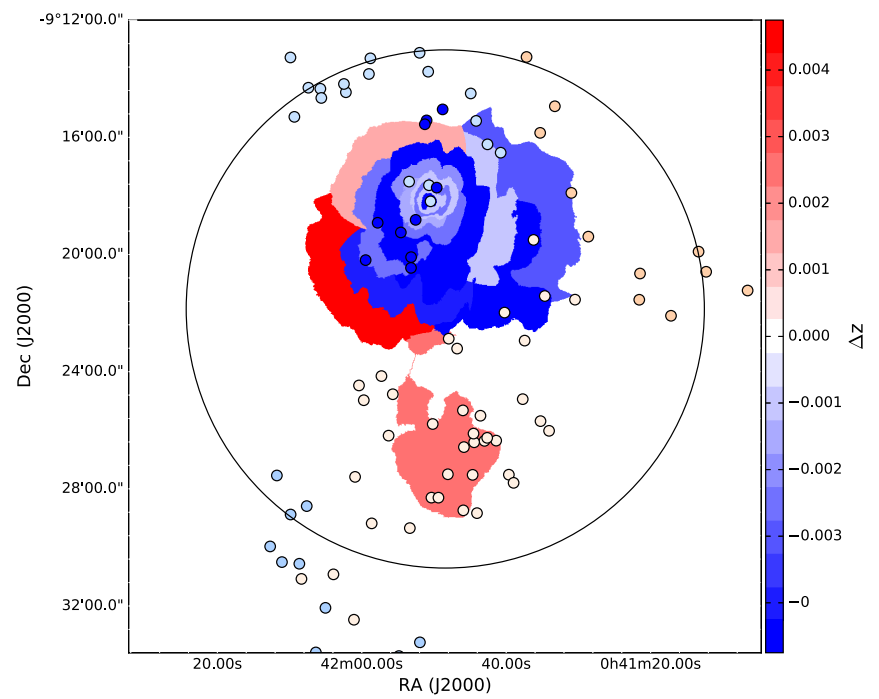

Figure 9. Redshift deviation map of the ICM of A85 with respect to its average redshift $\left(z_{\text {avg }}=0.0554\right)$. The bin size of the colored bar is $\delta z=0.001$. The galaxies (circles) are colored with the same redshift scale.

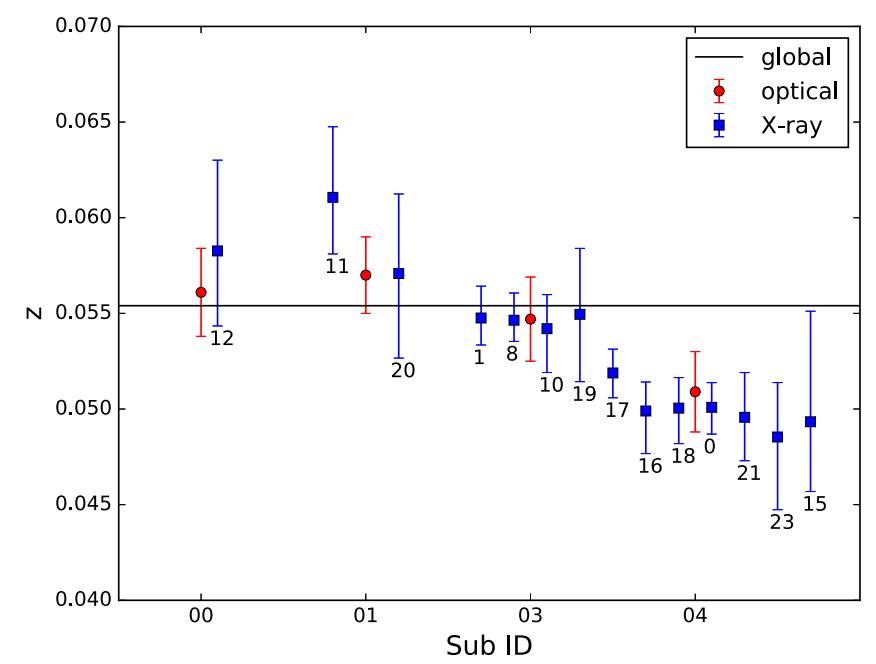

Figure 10. Redshifts of the optical substructures of A85 (red dots) and redshifts of the X-ray regions (blue squares). The abscissas of the blue squares are chosen according to their supposedly correlated optical substructures. The black solid line is the mean redshift $z_{\mathrm{avg}}=0.0554$.

in the central region of the cluster, identified with the third threshold on the binary tree of the caustic technique. We do not discuss sub02, because it is out of the Chandra field. The associations between galaxy substructures and ICM that we describe below are summarized in Figures 10 and 11.

The mean position of sub04 (cyan circles in Figure 11) overlaps with the central peak of the X-ray emission (Figure 8), with redshift $z=0.0509 \pm 0.0021$; the X-ray central regions ID 2, 13, 14, and 22 (yellow areas in Figure 11) have redshifts in the range 0.0532-0.0538. These redshifts appear substantially different from the sub04 redshift, suggesting that sub04 is now passing through the cluster core along the line of sight toward the observer at a velocity $v \sim c(0.0535-0.0509)=$ $780 \mathrm{~km} \mathrm{~s}^{-1}$, dragging some of the core X-ray gas, as indicated by the redshift of ID $0, z=0.0501_{-0.0013}^{+0.0014}$, consistent with the redshift of sub04. The substructure sub04 is falling into A85 with its own gas that can be identified with the X-ray regions ID 15, 16, 17, 18, 21, and 23 (cyan areas in Figure 11), whose 


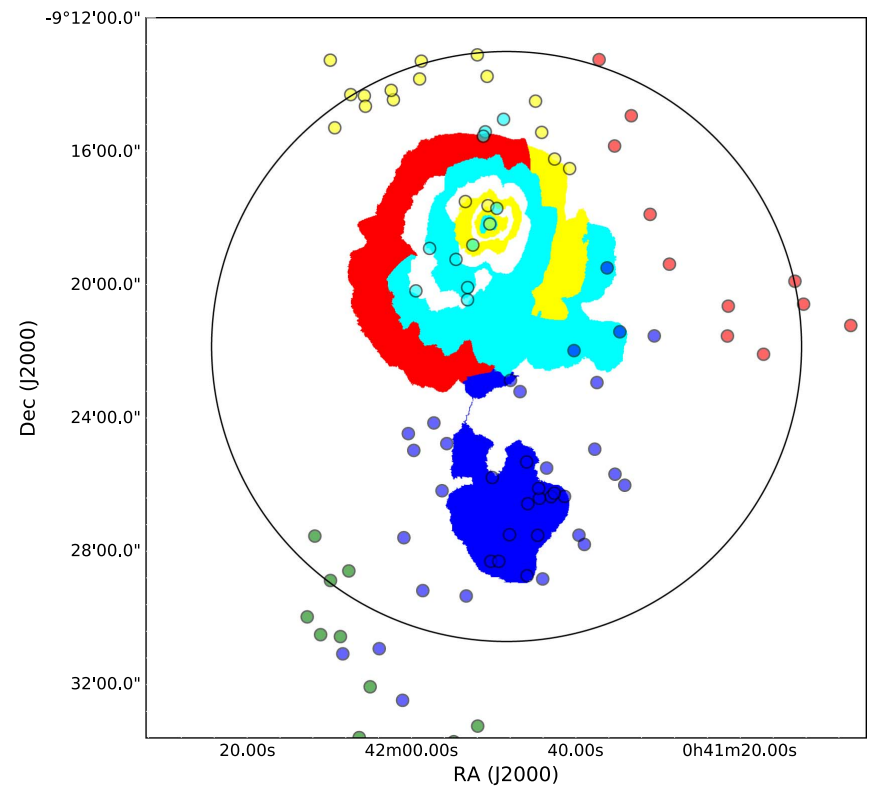

Figure 11. Spatial distribution of the associated optical substructures and the $\mathrm{X}$-ray regions. The related regions share the same color.

redshifts are in the range $0.0485-0.0519$, whereas some of its gas (ID $9, z=0.0527_{-0.0043}^{+0.0057}$ ) might be lagging behind because of ram pressure. In fact, galaxies are collisionless objects in cluster dynamics, and, during the cluster merging, they can easily move with the substructure of dark matter and separate from the baryonic gas (Markevitch et al. 2004; Bradač et al. 2008; Merten et al. 2011; Dawson et al. 2012; Dahle et al. 2013; Gastaldello et al. 2014).

Another group, sub03 (yellow circles in Figure 11), with redshift $z=0.0547 \pm 0.0022$, is going through the cluster from SW (bottom right of the plot of Figure 11) to NE (top left of the plot) almost perpendicularly to the line of sight. Unlike sub04, sub03 is not going exactly through the cluster core. Similarly to sub04 however, sub03 is moving with its own gas, associated with the X-ray regions ID 1, 8, 10, and 19 (yellow areas in Figure 11), with redshifts in the range 0.0542-0.0549, and it might be leaving behind some gas associated with ID 6 $\left(z=0.0556_{-0.0039}^{+0.0050}\right)$. The $\mathrm{BCG}$, whose redshift is $z=0.0554 \pm 0.0002$, belongs to sub03 and is located at the end of a plume of the X-ray temperature map recently unveiled (Ichinohe et al. 2015), suggesting that it is indeed moving through the cluster, exactly like sub03.

Sub01 (red circles in Figure 11), at redshift $z=0.0570 \pm 0.0020$, which is looser and poorer than the other substructures, also appears to be going through the cluster from $\mathrm{E}$ (left of the plot of Figure 11) to W (right of the plot) and is leaving behind some of its own gas associated with the region ID 20 (part of the red area in Figure 11), with $z=0.0571_{-0.0042}^{+0.0044}$, and probably also the region ID 11 (part of the red area in Figure 11), with $z=0.0611_{-0.0037}^{+0.0030}$, if sub01 has a non-negligible velocity component toward the observer.

Sub00 (blue circles in Figure 11) is the largest substructure in the cluster, covers a large area, and overlaps with two X-ray sub-peaks. Its redshift, $z=0.0561 \pm 0.0023$, is consistent with the redshift of the X-ray sub-peak associated to region ID 12 (blue area in Figure 11), $z=0.0583_{-0.0047}^{+0.0039}$.

\section{DISCUSSION AND CONCLUSION}

For the first time, we combine two techniques, the caustic technique based on optical spectroscopic redshifts and the $\mathrm{X}$-ray redshift fitting procedure, to explore the complex redshift structures of the central region of a galaxy cluster, A85, within $r_{500} \sim 0.6 \mathrm{~h}^{-1} \mathrm{Mpc}$, where the two probes provide overlapping data.

The substructures in the galaxy distribution and the ICM inhomogeneities observed in the X-ray band are correlated with each other, although they do not always share the same redshift and position on the sky. Galaxies and ICM have different evolving timescale, and are usually in different phases during a merging event.

We identify five substructures in A85 within $\sim 600 \mathrm{~h}^{-1} \mathrm{kpc}$ from the cluster X-ray peak: two substructures, sub01 and sub03, appear to have been recently accreted, and a substructure, sub00, is being currently accreted; the last substructure, sub04, with an optical redshift $z=0.0509$, has just gone through the cluster core almost exactly along the line of sight and is now moving out of the cluster toward the observer. Its existence is the main reason why previous investigations, both in the optical and X-ray, measured a cluster redshift smaller than the average redshift $z_{\text {avg }}=0.0554$. We conclude that A85 is not a relaxed system but has experienced recent merging events, in agreement with other investigations (e.g., Ichinohe et al. 2015). It will be interesting to investigate whether an accurate $N$-body/hydrodynamic simulation of the cluster mass accretion can reproduce the optical and X-ray kinematic properties of A85 that we find here.

Our analysis rests on a sample of optical redshifts mostly $(\sim 85 \%)$ derived from low-resolution spectra with $\sim 500 \mathrm{~km} \mathrm{~s}^{-1}$ uncertainty. This uncertainty is comparable to the cluster velocity dispersion $692_{-45}^{+55} \mathrm{~km} \mathrm{~s}^{-1}$ (Rines \& Diaferio 2006) and might affect the solidity of our substructure identification.

Yu et al. (2015) show that analyses of mock catalogs with perfectly known redshifts, based on the caustic technique with the automatic plateau and threshold identifications, return substructure catalogs that are $\sim 60 \%$ complete and contain $\sim 50 \%$ spurious substructures; Yu et al. (2015) also suggest that these results can improve when the binary tree threshold is tuned by hand, as we do here.

We tested our results as follows: we create synthetic redshift samples by replacing each individual redshift with a random variate extracted from a Gaussian probability density distribution with its mean set by the measured redshift and its dispersion set by the redshift uncertainty. Although the substructures identified in these synthetic samples might differ from sample to sample, we can still infer the same general picture: specifically we always find substructures associated with the X-ray filament and sub-peaks. The existence of the substructures called here sub03 and sub04 requires to be confirmed by more precise redshifts derived from highresolution optical spectra. Finally, the uncertainties of the $\mathrm{X}$-ray redshifts, which are the smallest uncertainties that can be obtained with current instrumentation, are $\sim 400 \mathrm{~km} \mathrm{~s}^{-1}$ or larger; therefore, our comparison is based on optical and X-ray data with comparable uncertainty, but clearly more robust results can only be reached by improving both optical observations and X-ray technology.

There are some uncertainties and limitations both in the substructure identification with the caustic method and in the $\mathrm{X}$-ray spectrum fitting procedure. Even with perfectly known 
individual galaxy redshifts, projection effects unavoidably weaken the solidity of the identification of the substructures with the caustic method and the estimate of their properties and their uncertainties, including their mean redshifts. On the other hand, the energy resolution of the X-ray detectors are limited and it appears hard to improve the precision of the redshift measurement with current devices.

Before the advent of a bolometer with high angular resolution, similar to what is planned for the upcoming X-ray telescope ATHENA (Barret et al. 2013), that will certainly provide a dramatic improvement in the field, an intermediate mission with instrumental features similar to the unfortunate X-ray observatory Hitomi (Astro-H, Takahashi et al. 2010) will be invaluable for the detailed investigation of the dynamics and thermodynamics of the ICM (Hitomi Collaboration et al. 2016).

When extended to a large cluster sample, the combined analysis of dense optical redshift surveys with those improved $\mathrm{X}$-ray spectroscopy from future X-ray telescopes, will certainly provide an essential contribution to our understanding of the growth history of galaxy clusters.

We sincerely thank Margaret Geller, Ana Laura Serra, and Jubee Sohn for numerous fruitful discussions. We acknowledge support from the grant Progetti di Ateneo/CSP_TO_Call2_2012_0011 "Marco Polo" of the University of Torino; the INFN grant InDark; the grant PRIN 2012 "Fisica Astroparticellare Teorica" of the Italian Ministry of University and Research; the National Natural Science Foundation of China under grant Nos. 11373014, 11073005, and 11403002; and the Fundamental Research Funds for the Central Universities and Scientific Research Foundation of Beijing Normal University. This work has been partially funded by the MINECO (grant AYA2013-43188-P). This research has made use of the Sixth Data Release of SDSS and the NASA/IPAC Extragalactic Database which is operated by the Jet Propulsion Laboratory, California Institute of Technology, under contract with the National Aeronautics and Space Administration. The WHT and its service program are operated on the island of La Palma by the Isaac Newton Group in the Spanish Observatorio del Roque de los Muchachos of the Instituto de Astrofísica de Canarias. I.A. and J.A.L.A. acknowledge support from the Ministerio de Economia y Competitividad (MINECO) under the grant AYA2013-43188-P. P.T. is supported by the Recruitment Program of High-end Foreign Experts and he gratefully acknowledges the hospitality of Beijing Normal University.

\section{REFERENCES}

Abazajian, K., Adelman-McCarthy, J. K., Agüeros, M. A., et al. 2003, AJ, 126,2081

Abazajian, K. N., Adelman-McCarthy, J. K., Agüeros, M. A., et al. 2009, ApJS, 182, 543

Abell, G. O., Corwin, H. G., Jr., \& Olowin, R. P. 1989, ApJS, 70, 1

Adelman-McCarthy, J. K., Agüeros, M. A., Allam, S. S., et al. 2008, ApJS, 175,297

Aguerri, J. A. L., \& Sánchez-Janssen, R. 2010, A\&A, 521, A28

Aguerri, J. A. L., Sánchez-Janssen, R., \& Muñoz-Tuñón, C. 2007, A\&A, 471,17

Agulli, I., Aguerri, J. A. L., Dominguez Palmero, L., \& Diaferio, A. 2016a, MNRAS, 461, L6

Agulli, I., Aguerri, J. A. L., Sánchez-Janssen, R., et al. 2014, MNRAS, 444, L34

Agulli, I., Aguerri, J. A. L., Sánchez-Janssen, R., et al. 2016b, MNRAS, 458, 1590

Ahn, C. P., Alexandroff, R., Allende Prieto, C., et al. 2014, ApJS, 211, 17
Andernach, H., Tago, E., Einasto, M., Einasto, J., \& Jaaniste, J. 2005, in ASP Conf. Ser. 329, Nearby Large-Scale Structures and the Zone of Avoidance, ed. A. P. Fairall \& P. A. Woudt (San Francisco, CA: ASP), 283

Arnaud, K. A. 1996, in ASP Conf. Ser. 101, Astronomical Data Analysis Software and Systems V, ed. G. H. Jacoby \& J. Barnes (San Francisco, CA: ASP), 17

Asplund, M., Grevesse, N., \& Sauval, A. J. 2005, ASP Conf. Ser. 336, Cosmic Abundances as Records of Stellar Evolution and Nucleosynthesis, ed. T. G. Barnes, III \& F. N. Bash (San Francisco, CA: ASP), 25

Balestra, I., Mercurio, A., Sartoris, B., et al. 2016, ApJS, 224, 33

Barret, D., Nandra, K., Barcons, X., et al. 2013, in Proc. Annual meeting of the French Society of Astronomy and Astrophysics SF2A-2013, ed. L. Cambresy et al. (Paris: Association Francaise d'Astronomie), 447

Beers, T. C., Gebhardt, K., Forman, W., Huchra, J. P., \& Jones, C. 1991, AJ, 102,1581

Beers, T. C., \& Geller, M. J. 1983, ApJ, 274, 491

Bœhm, C., Schewtschenko, J. A., Wilkinson, R. J., Baugh, C. M., \& Pascoli, S. 2014, MNRAS, 445, L31

Bond, J. R., Kofman, L., \& Pogosyan, D. 1996, Natur, 380, 603

Boué, G., Durret, F., Adami, C., et al. 2008, A\&A, 489, 11

Bourdin, H., Arnaud, M., Mazzotta, P., et al. 2011, A\&A, 527, A21

Bradač, M., Allen, S. W., Treu, T., et al. 2008, ApJ, 687, 959

Bravo-Alfaro, H., Caretta, C. A., Lobo, C., Durret, F., \& Scott, T. 2009, A\&A, 495, 379

Colberg, J. M., Krughoff, K. S., \& Connolly, A. J. 2005, MNRAS, 359, 272

Colless, M., Dalton, G., Maddox, S., et al. 2001, MNRAS, 328, 1039

Dahle, H., Sarazin, C. L., Lopez, L. A., et al. 2013, ApJ, 772, 23

Dawson, W. A., Wittman, D., Jee, M. J., et al. 2012, ApJL, 747, L42

de Lapparent, V., Geller, M. J., \& Huchra, J. P. 1986, ApJL, 302, L1

Del Popolo, A., \& Le Delliou, M. 2016, arXiv:1606.07790

Diaferio, A. 1999, MNRAS, 309, 610

Diaferio, A., \& Geller, M. J. 1997, ApJ, 481, 633

Dressler, A., \& Shectman, S. A. 1988, AJ, 95, 985

Durret, F., Felenbok, P., Lobo, C., \& Slezak, E. 1998, A\&AS, 129, 281

Durret, F., Lima Neto, G. B., \& Forman, W. 2005, A\&A, 432, 809

Durret, F., Lima Neto, G. B., Forman, W., \& Churazov, E. 2003, A\&A, 403, L29

Eckert, D., Jauzac, M., Shan, H., et al. 2015, Natur, 528, 105

Gastaldello, F., Limousin, M., Foëx, G., et al. 2014, MNRAS, 442, L76

Geller, M. J., \& Beers, T. C. 1982, PASP, 94, 421

Geller, M. J., Diaferio, A., Rines, K. J., \& Serra, A. L. 2013, ApJ, 764, 58

Geller, M. J., \& Huchra, J. P. 1989, Sci, 246, 897

Geller, M. J., Hwang, H. S., Diaferio, A., et al. 2014, ApJ, 783, 52

Girardi, M., Boschin, W., Gastaldello, F., et al. 2016, MNRAS, 456, 2829

Girardi, M., Mercurio, A., Balestra, I., et al. 2015, A\&A, 579, A4

Grillo, C., Suyu, S. H., Rosati, P., et al. 2015, ApJ, 800, 38

Guennou, L., Adami, C., Durret, F., et al. 2014, A\&A, 561, A112

Harvey, D., Massey, R., Kitching, T., Taylor, A., \& Tittley, E. 2015, Sci, 347,1462

Hess, K. M., Jarrett, T. H., Carignan, C., Passmoor, S. S., \& Goedhart, S. 2015, MNRAS, 452, 1617

Hitomi Collaboration, Aharonian, F., Akamatsu, H., et al. 2016, Natur, 535,117

Hoekstra, H., Franx, M., \& Kuijken, K. 2000, ApJ, 532, 88

Hwang, H. S., Geller, M. J., Diaferio, A., \& Rines, K. J. 2012, ApJ, 752, 64

Hwang, H. S., Geller, M. J., Diaferio, A., Rines, K. J., \& Zahid, H. J. 2014 ApJ, 797, 106

Hwang, H. S., Geller, M. J., Park, C., et al. 2016, ApJ, 818, 173

Ichinohe, Y., Werner, N., Simionescu, A., et al. 2015, MNRAS, 448, 2971

Jauzac, M., Eckert, D., Schwinn, J., et al. 2016, arXiv:1606.04527

Jauzac, M., Jullo, E., Eckert, D., et al. 2015, MNRAS, 446, 4132

Jeltema, T. E., Canizares, C. R., Bautz, M. W., \& Buote, D. A. 2005, ApJ, 624,606

Jonathan Goodman, J. W. 2010, Communications in Applied Mathematics and Computational Science, 5, 65

Kaastra, J. 1992, An X-Ray Spectral Code for Optically Thin Plasmas (Internal SRONLeiden Report, updated version 2.0)

Kahlhoefer, F., Schmidt-Hoberg, K., Frandsen, M. T., \& Sarkar, S. 2014, MNRAS, 437, 2865

Kneib, J.-P., Ellis, R. S., Smail, I., Couch, W. J., \& Sharples, R. M. 1996, ApJ, 471,643

Kolokotronis, V., Basilakos, S., Plionis, M., \& Georgantopoulos, I. 2001, MNRAS, 320, 49

Lauer, T. R., Postman, M., Strauss, M. A., Graves, G. J., \& Chisari, N. E. 2014, ApJ, 797, 82 
Lee, G.-H., Hwang, H. S., Lee, M. G., et al. 2015, ApJ, 800, 80 Liedahl, D. A., Osterheld, A. L., \& Goldstein, W. H. 1995, ApJL, 438, L115 Lin, Y.-T., \& Mohr, J. J. 2004, ApJ, 617, 879

Liu, A., Yu, H., Tozzi, P., \& Zhu, Z.-H. 2015, ApJ, 809, 27

Liu, A., Yu, H., Tozzi, P., \& Zhu, Z.-H. 2016, ApJ, 821, 29

Markevitch, M., Gonzalez, A. H., Clowe, D., et al. 2004, ApJ, 606, 819

Massey, R., Williams, L., Smit, R., et al. 2015, MNRAS, 449, 3393

Medezinski, E., Umetsu, K., Nonino, M., et al. 2013, ApJ, 777, 43

Merten, J., Coe, D., Dupke, R., et al. 2011, MNRAS, 417, 333

Mewe, R., Gronenschild, E. H. B. M., \& van den Oord, G. H. J. 1985, A\&AS 62, 197

Mewe, R., Lemen, J. R., \& van den Oord, G. H. J. 1986, A\&AS, 65, 511

Miller, C. J., Nichol, R. C., Reichart, D., et al. 2005, AJ, 130, 968

Mohr, J. J., Fabricant, D. G., \& Geller, M. J. 1993, ApJ, 413, 492

Mohr, J. J., Geller, M. J., \& Wegner, G. 1996, AJ, 112, 1816

Oegerle, W. R., \& Hill, J. M. 2001, AJ, 122, 2858

Ogrean, G. A., van Weeren, R. J., Jones, C., et al. 2015, ApJ, 812, 153

Ogrean, G. A., van Weeren, R. J., Jones, C., et al. 2016, ApJ, 819, 113

Okabe, N., Futamase, T., Kajisawa, M., \& Kuroshima, R. 2014, ApJ, 784, 90

Parekh, V., van der Heyden, K., Ferrari, C., Angus, G., \& Holwerda, B. 2015, A\&A, 575, A127

Pranger, F., Böhm, A., Ferrari, C., et al. 2013, A\&A, 557, A62

Pranger, F., Böhm, A., Ferrari, C., et al. 2014, A\&A, 570, A40

Ramella, M., Biviano, A., Pisani, A., et al. 2007, A\&A, 470, 39
Rines, K., \& Diaferio, A. 2006, AJ, 132, 1275

Rines, K., Mahdavi, A., Geller, M. J., et al. 2001, ApJ, 555, 558

Robertson, A., Massey, R., \& Eke, V. 2016, arXiv:1605.04307

Rossetti, M., Gastaldello, F., Ferioli, G., et al. 2016, MNRAS, 457, 4515

Sanders, J. S. 2006, MNRAS, 371, 829

Schenck, D. E., Datta, A., Burns, J. O., \& Skillman, S. 2014, AJ, 148, 23

Serra, A. L., \& Diaferio, A. 2013, ApJ, 768, 116

Serra, A. L., Diaferio, A., Murante, G., \& Borgani, S. 2011, MNRAS, 412, 800

Smith, R. J., Hudson, M. J., Nelan, J. E., et al. 2004, AJ, 128, 1558

Spergel, D. N., \& Steinhardt, P. J. 2000, PhRvL, 84, 3760

Struble, M. F., \& Rood, H. J. 1991, ApJS, 77, 363

Takahashi, T., Mitsuda, K., Kelley, R., et al. 2010, Proc. SPIE, 7732, 0

Tanaka, N., Furuzawa, A., Miyoshi, S. J., Tamura, T., \& Takata, T. 2010, PASJ, 62, 743

Utsumi, Y., Geller, M. J., Dell'Antonio, I. P., et al. 2016, arXiv:1606.07439

Viel, M., Becker, G. D., Bolton, J. S., \& Haehnelt, M. G. 2013, PhRvD, 88, 043502

Wilms, J., Allen, A., \& McCray, R. 2000, ApJ, 542, 914

Yu, H., Serra, A. L., Diaferio, A., \& Baldi, M. 2015, ApJ, 810, 37

Yu, H., Tozzi, P., Borgani, S., Rosati, P., \& Zhu, Z.-H. 2011, A\&A, 529, A65

Zhang, Y.-Y., Okabe, N., Finoguenov, A., et al. 2010, ApJ, 711, 1033

Zitrin, A., Bartelmann, M., Umetsu, K., Oguri, M., \& Broadhurst, T. 2012, MNRAS, 426, 2944 